\title{
Car Delay Model near Bus Stops with Mixed Traffic Flow
}

\author{
Yang Xiaobao, Huan Mei, and Gao Ziyou \\ MOE Key Laboratory for Urban Transportation Complex Systems Theory and Technology, \\ Beijing Jiaotong University, Beijing 100044, China \\ Correspondence should be addressed to Yang Xiaobao; yangxb@bjtu.edu.cn
}

Received 27 January 2013; Accepted 23 April 2013

Academic Editor: Xiaoning Zhang

Copyright (C) 2013 Yang Xiaobao et al. This is an open access article distributed under the Creative Commons Attribution License, which permits unrestricted use, distribution, and reproduction in any medium, provided the original work is properly cited.

This paper proposes a model for estimating car delays at bus stops under mixed traffic using probability theory and queuing theory. The roadway is divided to serve motorized and nonmotorized traffic streams. Bus stops are located on the nonmotorized lanes. When buses dwell at the stop, they block the bicycles. Thus, two conflict points between car stream and other traffic stream are identified. The first conflict point occurs as bicycles merge to the motorized lane to avoid waiting behind the stopping buses. The second occurs as buses merge back to the motorized lane. The average car delay is estimated as the sum of the average delay at these two conflict points and the delay resulting from following the slower bicycles that merged into the motorized lane. Data are collected to calibrate and validate the developed model from one site in Beijing. The sensitivity of car delay to various operation conditions is examined. The results show that both bus stream and bicycle stream have significant effects on car delay. At bus volumes above 200 vehicles per hour, the curbside stop design is not appropriate because of the long car delays. It can be replaced by the bus bay design.

\section{Introduction}

As the first point of contact between the passenger and the public transit service, the bus stop is a critical element in a transit system's overall goal of providing timely, safe, and convenient transportation. In the past several decades, traffic planners, designers, and scholars have paid much attention to the location, design, and operations of bus stops [1-5]. A prominent achievement of this research is a set of guidelines for use in designing and locating bus stops, sponsored by TCRP in the United States [1]. Other researchers mainly focused on the effects of bus stops on traffic flow. For example, Wong et al. analyzed the delay at a signal-controlled intersection with a bus stop upstream [6]. Fernández applied the microscopic traffic simulation model to study operational impacts on bus stops such as capacity, delays, queues, and waiting times [7]. Tang et al. used the macrodynamic model to analyze the effects of bus stop on traffic flow [8]. Most existing research of bus stops analyzes only the mixed traffic flow between buses and cars without including nonmotorized vehicles. This might be the reason that the car-bus conflict at bus stops is usually regarded as more important than the motorized vehicle-bicycle conflict. Another reason might be the low proportion of cycling among all travel modes in developed countries.

As a developing country, China has its own traffic characteristics. A mix of nonmotorized and motorized vehicles is an important traffic type in China. Some surveys show that the nonmotorized vehicle, especially the bicycle, is one of the most widely used traffic tools in Chinese daily travel activity. Typically, there are three types of bus stops in urban areas: curbside stops, bus bays, and bus boarders [9]. The curbside stop is the most common type on minor roadways in many Chinese cities. Figure 1 shows the mixed traffic streams at a typical curbside stop. There are two lanes on the urban roadway: the bicycle lane and the motorized lane, and there are three types of traffic streams: bicycle, bus, and car. Bus stops are usually located on the bicycle lane. When a bus dwells at the curbside stop, it blocks the bicycles. Bicycles merge to the motorized lane to avoid waiting behind the stopping bus. Thus, the presence of a stopped bus creates a temporary conflict between bicycles and cars. In addition, the car-bus conflict takes place when a bus departs from the stop to the motorized lane. Similar phenomena may be found in other Asian developing countries, for example, India, Malaysia, Vietnam, and Cambodia. 


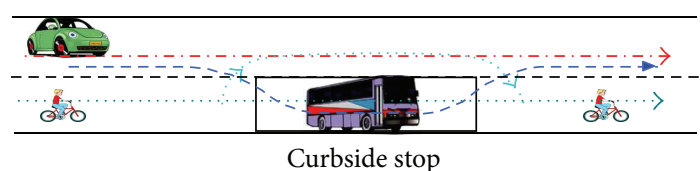

FIGURE 1: Curbside stop with mixed traffic streams of buses, cars, and bicycles.

Due to the special features of mixed traffic, the application of existing traffic models for bus stops, developed by developed countries, has not produced a clear effect on Chinese traffic management and control. Therefore, it is necessary to deeply study the mixed traffic flow between nonmotorized vehicles and motorized vehicles.

On the mixed traffic, till now, much research has been conducted on basic segments and intersections [10, 11], but the correlative research on bus stops is much less in the literature. In recent years, some researchers have realized this, and correlative work is being done, but it still has a long way to go. Koshy and Arasan used simulation technique to study the impact of bus stop type on the speeds of other vehicles under heterogeneous conditions [9]. Yang et al. established car capacity models near a curbside stop with bicycles based on gap acceptance theory and conflict technique [12, 13]. However, little information was found in the literature on delay time near bus stops with mixed traffic flow. This paper investigates car delay time near a curbside stop under mixed traffic conditions. Firstly, mixed traffic flow characteristics near bus stops are analyzed. Then, the delay model based on probability and queuing theory is proposed. Next, the delay model is validated by field data in Beijing. In addition, the sensitivity of car delay to various operation conditions is examined. Finally, conclusions and future researches are given.

\section{Mixed Traffic Flow Characteristics near Curbside Stops}

2.1. Bus Stream: $M / M / k$ Queuing Model. Consider a road link near the bus stop as shown in Figure 1. A sophisticated queuing theory model can be developed on the assumption that the simple bus stream system can be represented by an $\mathrm{M} / \mathrm{M} / k$ queue. The service counter is the bus stop. The input into the system in equilibrium, as well as the output, is formed by the buses approaching from upstream, which are assumed to arrive at random; that is, there are negative exponentially distributed arrival headways with mean $1 / \lambda_{b}$ seconds. The dwelling time at the stop is the service time, which is also assumed to be independent and negative exponential distributed random variables with mean $t_{b}$ seconds. Finally, the " $k$ " in $\mathrm{M} / \mathrm{M} / k$ stands for $k$ identical servers, that is, the number of existing berths at the bus stop.

For the $\mathrm{M} / \mathrm{M} / k$ system as a general property, the probability of the busy system is given by the following equation. That is, the probability of one or more buses at the stop is

$$
p_{s}=1-\left(\sum_{j=0}^{k-1} \frac{\rho_{b}^{j}}{j !}+\frac{\rho_{b}^{k}}{k !} \cdot \frac{k}{k-\rho_{b}}\right)^{-1} .
$$

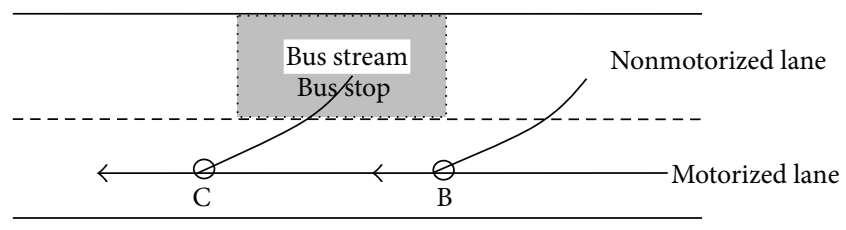

FIGURE 2: Conflict among cars, buses and bicycles at a curbside stop.

Note that, here, $\rho_{b}=\lambda_{b} t_{b}$, and for the existence of a steady-state solution, $\lambda_{b}<k / t_{b}$. The subscript " $b$ " stands for bus stream passing the stop and the subscript " $s$ " stands for bus stream at the stop.

And the expected number in the system at steady state; that is, the expected number of buses both in service and in queue at the stop is

$$
L=E(N)=\frac{\rho_{b}^{k}}{k !} \cdot \frac{k \rho_{b}}{\left(k-\rho_{b}\right)^{2}}\left(1-p_{s}\right)+\rho_{b}
$$

2.2. Conflict between Different Streams. At the curbside stop with mixed traffic flow, there are three streams among buses, bicycles, and cars. As shown in Figure 2, car stream is directly affected by two conflicts. One is the interaction between car stream and bicycle stream at point $\mathrm{B}$ when one or more buses dwell at the stop. The other is the conflict between car stream and bus stream at point $\mathrm{C}$ as buses merge back to the motorized lane.

When a bus dwells at the curbside stop in the nonmotorized lane, the nonmotorized lane is blocked by a stopped bus. A lane change for bicycles from the nonmotorized lane to the motorized lane is "essential" when bicyclists approach to the last stopped bus. As a result, forced lane changing maneuvers take place. Bicycles in the nonmotorized lane would force the subsequent car in the motorized lane to slow down for the lane-changing execution. Field observations indicate that this cooperative lane changing and priority-sharing behavior is prevalent between bicycles and cars near bus stops [13]. As the acceptable gap of bicycles is approximate to the follow-up time of successive cars, the bicycle-car conflict near a bus top is similar to the conflict at merges under low speed or high flow conditions. In the saturated traffic flow, gap acceptance theory completely loses its applicability; waiting vehicles generally perform forced lane-changing maneuvers and pass the conflict point alternately $[14,15]$. That is to say, they comply with the FIFO (first-in-first-out) discipline under low speed condition. Near a curbside stop, the vehicles usually pass the conflicting areas with a low speed because there is a serious conflict among different streams. The conflicting areas near a curbside stop can also be considered in such a way that the FIFO discipline is applied.

\section{Delay Model for Car Stream near the Stop under Mixed Traffic Flow}

The average delay to car stream near the stop under mixed traffic conditions is estimated as the sum of the average delay at these two conflict points and the delay resulting 
from following the slower bicycle traffic that merged into the motorized lanes.

3.1. Delay Resulting from following the Slower Bicycles from Point $B$ to Point $C$. Both car stream and bicycle stream are assumed to arrive at random, that is, negative exponentially distributed arrival headways. Let $t_{n}$ denote the time that a bicycle (nonmotorized vehicle) arrives at point $\mathrm{B}$, and $t_{c}$ denote the time that a car arrives at point $B$ behind the bicycle. Let $Z$ be a nonnegative random variable representing the difference between $t_{c}$ and $t_{n}$ :

$$
Z=t_{c}-t_{n}
$$

Then, the probability that $Z$ is not more than a given time $z$ can be expressed as

$$
\begin{aligned}
P(Z \leq z) & =\iint_{t_{c}-t_{n} \leq z} f\left(t_{n}\right) f\left(t_{c}\right) d t_{c} d t_{n} \\
& =\int_{0}^{+\infty} \int_{0}^{z+t_{n}} f\left(t_{n}\right) f\left(t_{c}\right) d t_{c} d t_{n} \\
& =\int_{0}^{+\infty} \int_{0}^{z+t_{n}} \lambda_{n} e^{-\lambda_{n} t_{n}} \cdot \lambda_{c} e^{-\lambda_{c} t_{c}} d t_{c} d t_{n} \\
& =1-\frac{\lambda_{n}}{\lambda_{n}+\lambda_{c}} e^{-\lambda_{c} z} .
\end{aligned}
$$

Then, the probability density function of $Z$ is

$$
f(z)=\frac{d F(z)}{d z}=\frac{d P(Z \leq z)}{d z}=\frac{\lambda_{n} \lambda_{c}}{\lambda_{n}+\lambda_{c}} e^{-\lambda_{c} z} .
$$

As shown in Figure 2, it may occur that a car follows the slower bicycles when a bicyclist rides at section BC. The distance from $\mathrm{B}$ to $\mathrm{C}$ can be obtained by the formula:

$$
l_{\mathrm{BC}}=l_{b} \cdot L,
$$

where $l_{b}$ is the minimum headway distance of successively stopped buses at the stop and $L$ is the expected number of buses at the stop which can be given as (2).

The phenomenon that a car follows the slower bicycle takes place only when one or more buses berth the stop and $Z$ falls within limits, $0 \leq Z \leq z_{\max }$. The minimum value, 0 , represents the condition that a car begins to follow a bicycle from point $\mathrm{B}$. The maximum value, $z_{\max }$, represents the condition that a car begins to follow a bicycle from point C. If $Z$ is less than $z_{\max }$, a car must decelerate to follow the preceding bicycle before point C. $z_{\max }$ can be calculated as

$$
z_{\max }=l_{\mathrm{BC}}\left(\frac{1}{v_{1 n}}-\frac{1}{v_{1 c}}\right),
$$

where $v_{1 c}$ and $v_{1 n}$ are the free-flow velocity for car stream and bicycle stream near a curbside stop, respectively. It is noted that $\left(1 / v_{1 n}-1 / v_{1 c}\right)$ is the delay for a car driving one meter when it decelerates to follow the slower bicycle.

Let a car with the free-flow velocity catch up with its preceding bicycle at the time $t$. In this case, the distance that the car drives from time $t_{c}$ to $t$ is equal to the distance that the bicyclist rides from time $t_{n}$ to $t$, which can be expressed as

$$
l_{1}=v_{1 n}\left(t-t_{n}\right)=v_{1 c}\left(t-t_{c}\right) .
$$

Combined with (3), (7), and (8), $l_{1}$ can be given as

$$
l_{1}=\frac{Z}{\left(1 / v_{1 n}-1 / v_{1 c}\right)}=Z \cdot \frac{l_{\mathrm{BC}}}{z_{\max }} .
$$

Thus, the remaining distance that a car must decelerate to follow its preceding bicycle in section $\mathrm{BC}$ is

$$
l_{2}=l_{\mathrm{BC}}-l_{1}=l_{\mathrm{BC}}-Z \cdot \frac{l_{\mathrm{BC}}}{z_{\max }} .
$$

The expected value that a car decelerates to follow the preceding bicycle can be calculated as

$$
\begin{aligned}
E\left(l_{2}\right)= & l_{\mathrm{BC}}-\frac{l_{\mathrm{BC}}}{z_{\max }} \int_{0}^{z_{\max }} z f(z) d z \\
= & l_{\mathrm{BC}}-\frac{\lambda_{n} l_{\mathrm{BC}}}{\left(\lambda_{n}+\lambda_{c}\right) \lambda_{c} z_{\max }} \\
& \times\left(1-e^{-\lambda_{c} z_{\max }}-\lambda_{c} z_{\max } e^{-\lambda_{c} z_{\max }}\right) .
\end{aligned}
$$

It occurs that a car follows the slower bicycles only when one or more buses berth the stop. Thus, combined with (1), (7), and (11), the delay resulting from following the slower bicycles can be calculated as

$$
\begin{aligned}
d_{\mathrm{BC}} & =p_{s} \cdot E\left(l_{2}\right) \cdot\left(\frac{1}{v_{1 n}}-\frac{1}{v_{1 c}}\right) \\
& =p_{s}\left[z_{\max }-\frac{\lambda_{n}}{\left(\lambda_{n}+\lambda_{c}\right) \lambda_{c}}\left(1-e^{-\lambda_{c} z_{\max }}-\lambda_{c} z_{\max } e^{-\lambda_{c} z_{\max }}\right)\right] .
\end{aligned}
$$

3.2. Delay at Two Conflict Points. As shown in Figure 2, for the conflicting point $\mathrm{B}$, if one or more buses berth the stop, the car-bicycle conflict takes place. The conflicting areas near bus stops can be considered in such a way that the FIFO discipline is applied. Because different stream has different service time passing the conflicting area, the car-bicycle conflict at point $\mathrm{B}$ can be represented by the advanced Markovian model with no priorities but unequal arrival rates and unequal service rates for customers of two major types. Similarly, the carbus conflict at point $\mathrm{C}$ can be described by the advanced Markovian model.

It is assumed that each stream arrives as a Poisson process to a single exponential channel, and there are two types of customers with no priorities but unequal arrival rates $\left(\lambda_{1}, \lambda_{2}\right)$ and unequal service rates $\left(\mu_{1}, \mu_{2}\right.$, and $\left.\mu_{2}>\mu_{1}\right)$. Here, service rate $(\mu)$ is the reciprocal value of the mean service time $(s)$, that is, $\mu_{1}=1 / s_{1}, \mu_{2}=1 / s_{2}$. Then, the expected waiting time for each type of customers in queue at steady state [16] is

$$
\begin{array}{r}
W_{q_{1}}=\frac{\left(\lambda_{1}+\lambda_{2}\right) s_{1}^{2}\left[1-\lambda_{2} s_{2}\left(1-s_{2} / s_{1}\right)\right]}{\left(1-\lambda_{1} s_{1}-\lambda_{2} s_{2}\right)}, \\
W_{q_{2}}=\frac{\left(\lambda_{1}+\lambda_{2}\right) s_{1}^{2}\left[s_{2}^{2} / s_{1}^{2}+\left(1-s_{2} / s_{1}\right)\left(\lambda_{1} s_{2}\right)\right]}{\left(1-\lambda_{1} s_{1}-\lambda_{2} s_{2}\right)} .
\end{array}
$$


3.2.1. Delay Resulting from the Car-Bicycle Conflict at Point B. Traffic conditions near a curbside stop are classified into two types: inexistence and existence of stopped bus at the stop. The probabilities of these two conditions can be obtained by using (1). Under the former condition, the bicycle stream and the car stream at point B have no conflict and car travel time is not affected by the bicycle stream. Under the latter condition, the car-bicycle conflict at point B leads to an effect on car travel time by the bicycle stream. In this case, the carbicycle conflict at point $\mathrm{B}$ can be represented by the advanced Markovian model. The car delay caused by the car-bicycle conflict is equal to the expected waiting time in the queue system for mixed streams between cars and bicycles, $W_{c, \mathrm{~B}}$. As the mean service time for car stream at point $B$ is larger than that for bicycle stream, $W_{c, \mathrm{~B}}$ can be obtained by (13). Thus, the car delay at the point $\mathrm{B}$ can be given as

$$
\begin{aligned}
d_{\mathrm{B}} & =p_{s} W_{c, \mathrm{~B}}+\left(1-p_{s}\right) \cdot 0 \\
& =p_{s} s_{c}^{2}\left(\lambda_{c}+\lambda_{n}\right) \frac{1-\left(1-s_{n} / s_{c}\right) \lambda_{n} s_{n}}{1-\lambda_{c} s_{c}-\lambda_{n} s_{n}},
\end{aligned}
$$

where $\lambda_{n}$ and $\lambda_{c}$ are the arrival rate of bicycle stream and car stream approaching the conflicting point $\mathrm{B}$, respectively. $s_{n}$ and $s_{c}$ are the mean service time of bicycle stream and car stream passing the point $B$, respectively.

3.2.2. Delay Resulting from the Car-Bus Conflict at Point C. As buses merge back to the motorized lane, the car-bus conflict takes place. The car-bus conflict at point $\mathrm{C}$ can be represented by the advanced Markovian model. The car delay caused by the car-bus conflict is equal to the expected waiting time in the queue system for mixed streams between cars and buses, $W_{c, \mathrm{C}}$. As the mean service time for car stream at point $\mathrm{C}$ is less than that for bus stream, $W_{c, \mathrm{C}}$ can be obtained by (14). Thus, the car delay at the point $\mathrm{C}$ can be given as

$$
d_{\mathrm{C}}=W_{c, \mathrm{C}}=s_{b}^{\prime 2}\left(\lambda_{c}+\lambda_{b}\right) \frac{s_{c}^{2} / s_{b}^{\prime 2}+\left(1-s_{c} / s_{b}^{\prime}\right) \lambda_{b} s_{c}}{1-\lambda_{b} s_{b}^{\prime}-\lambda_{c} s_{c}},
$$

where $\lambda_{b}$ and $\lambda_{c}$ are the arrival rate of bus stream and car stream, respectively. $s_{b}^{\prime}$ and $s_{c}$ are the mean service time of bus stream and car stream passing the point $\mathrm{C}$, respectively.

\section{Model Validation and Comparison}

In order to calibrate the proposed model of car delay at the curbside stop under mixed traffic conditions, field data collected at a bus stop in Beijing were employed. Video cameras were used to record traffic operations at the bus stop. Vehicle type, flows, and travel times were recorded for each vehicle passing through the stop. In addition, the dwell time of bus stream and the headways in the conflicting area were also recorded. Data were collected in the spring of 2008 in one direction over 3 minutes categorized into a group.

The basic parameter used to compute car delay at a stop under mixed traffic is the service time for each stream. The service time was directly measured for each vehicle at the conflicting point using video during this study. The service

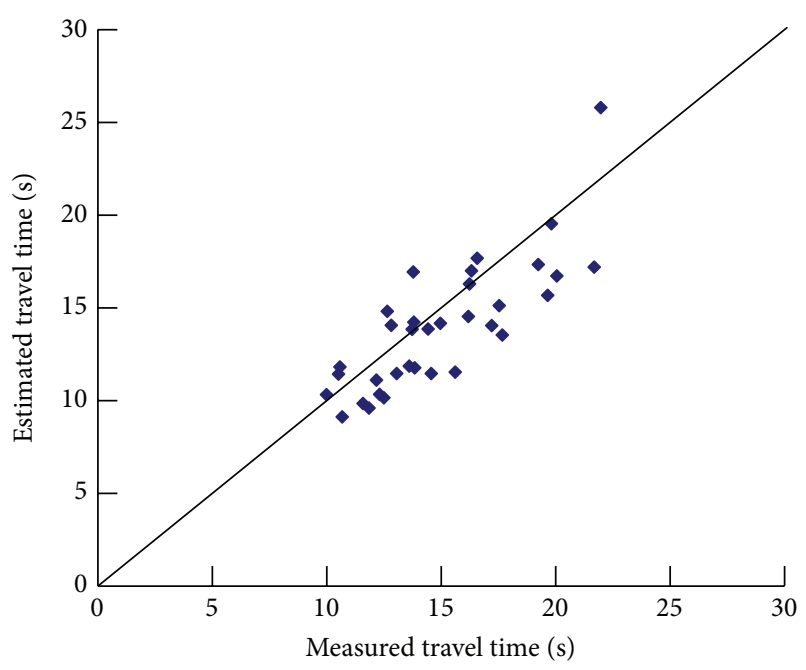

FIGURE 3: Comparison of measured average travel time and estimated average travel time.

time in this paper is the follow-up headway for vehicles in this approach if no vehicle is waiting on the conflicting approach and is equal to the minimum saturation headway. On the basis of field survey and video process, the minimum saturation headways for bicycle stream, car stream, and bus stream are $0.90 \mathrm{~s}, 2.04 \mathrm{~s}$, and $4.27 \mathrm{~s}$, respectively. Here, the relatively low value for bicycle stream is the result of cycling parallel behavior and group behavior. In addition, $l_{b}=12 \mathrm{~m}$, $v_{1 n}=4.5 \mathrm{~m} / \mathrm{s}$, and $v_{1 c}=10 \mathrm{~m} / \mathrm{s}$ on the surveyed curbside stops in Beijing.

The data collected in field study are used to validate the model, as shown in Figure 3. To facilitate comparison, the line where the measured average travel time equals to the estimated average travel time is superimposed on each figure. And it is found that scatter dots fluctuates narrowly around the line. In addition, the mean percent error between the estimated travel times and the measured times is $-6.6 \%$, and the mean absolute percentage error is $12.7 \%$. Thus, the proposed delay model at the stop with mixed traffic flow is desirable. Before the applications, however, it is noted that the model should be estimated using the specified field data.

\section{Effects of Individual Traffic Stream on Car Delay}

Differences in the arrival rate of bicycle stream affect car delay time near the curbside stop are shown in Figure 4. Here, the curbside stop has two berths; that is to say, bus stream system can be considered as an $\mathrm{M} / \mathrm{M} / 2$ queue. In addition, $\lambda_{b}=0.03 \mathrm{veh} / \mathrm{s}, s_{b}=25 \mathrm{~s}, l_{b}=12 \mathrm{~m}, v_{1 n}=4.5 \mathrm{~m} / \mathrm{s}$, and $v_{1 c}=10 \mathrm{~m} / \mathrm{s}$, and the minimum saturation headways for bicycle stream, car stream, and bus stream are $0.90 \mathrm{~s}, 2.04 \mathrm{~s}$, and $4.27 \mathrm{~s}$, respectively. Bicycle stream headways follow the negative exponential distribution. At the same car flow rate, the probability of the car-bicycle conflict increases with the increasing bicycle stream, which finally lead to the increase of car delays. 


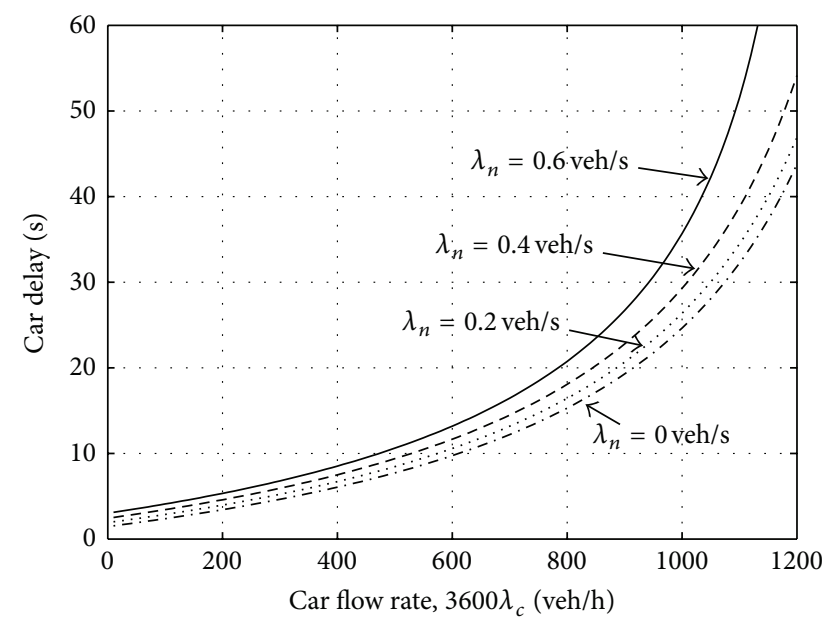

FIgURE 4: Car delay with different arrival rates for bicycle stream, $\lambda_{b}=0.03 \mathrm{veh} / \mathrm{s}$ and $s_{b}=25 \mathrm{~s}$.

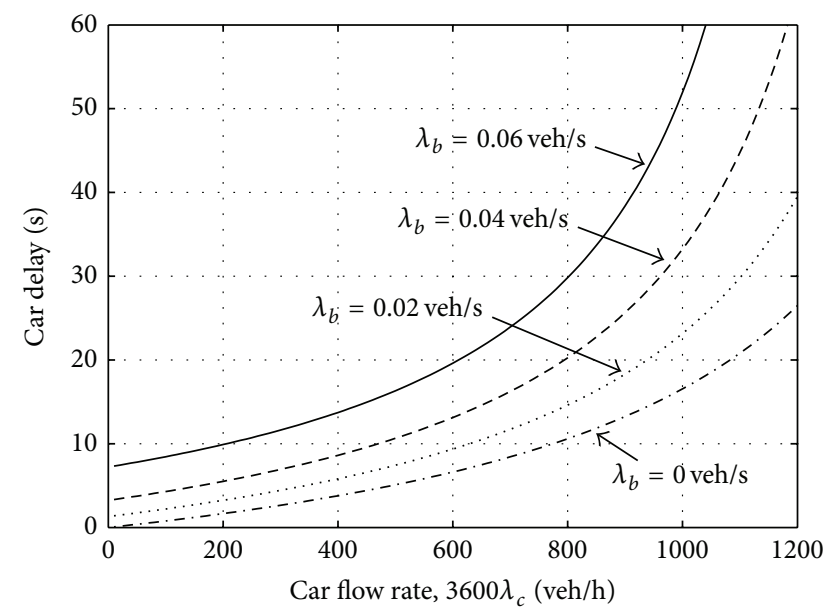

Figure 5: Car delay with different arrival rates for bus stream, $\lambda_{n}=$ $0.3 \mathrm{veh} / \mathrm{s}$ and $s_{b}=25 \mathrm{~s}$.

Figures 5 and 6 give the effects of bus stream on car delay time. Firstly, as shown in Figure 5, the probability of one or more stopped buses at the stop increases with the increasing bus flow rate, and so does also the probability of the carbus conflict. This extends car travel time. Similarly, Figure 6 displays car delay with different dwelling times of bus stream. As the dwelling time of bus stream increases, the probability of one or more stopped buses at the stop increases. This increases the delay time for car stream on the basis of (12) and (15).

In addition, Figures 4, 5, and 6 all show that car delay increases with the creasing car flow rate. Meanwhile, a comparison of these three figures indicates that bus flow rate has the most significant effect on car delays. This is because all parts of car delay (including $d_{\mathrm{B}}, d_{\mathrm{BC}}$, and $d_{\mathrm{C}}$ ) increase as bus flow rate increases. As shown in Figure 7, especially, at high bus flow rates, as the queuing systems are near the threshold values of equilibrium conditions; that is, $\lambda_{c} s_{c}+\lambda_{n} s_{n}$ or $\lambda_{b} s_{b} / k$ is near to one, $d_{\mathrm{B}}$ and $d_{\mathrm{BC}}$ will become infinite. The results

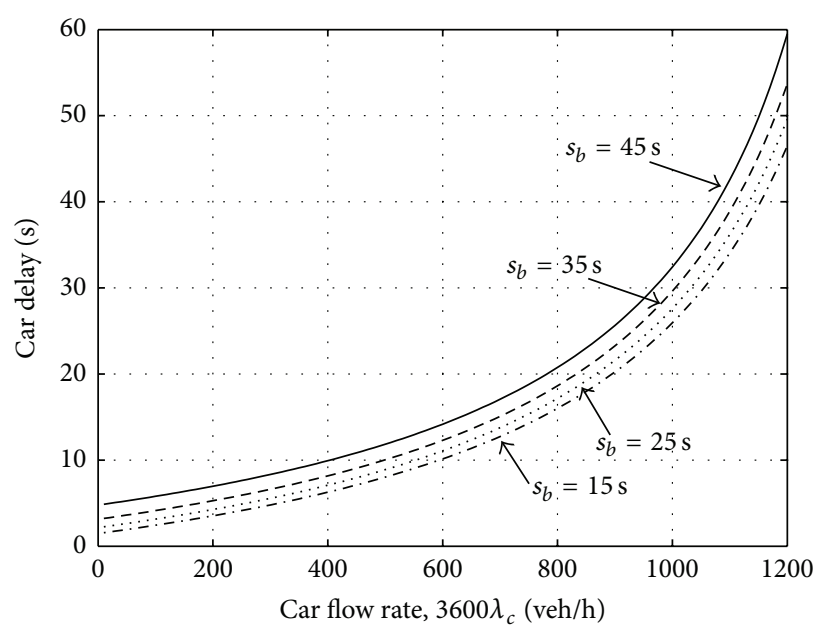

FIGURE 6: Car delay with different dwelling times for bus stream, $\lambda_{n}=0.3 \mathrm{veh} / \mathrm{s}$ and $\lambda_{b}=0.03 \mathrm{veh} / \mathrm{s}$.

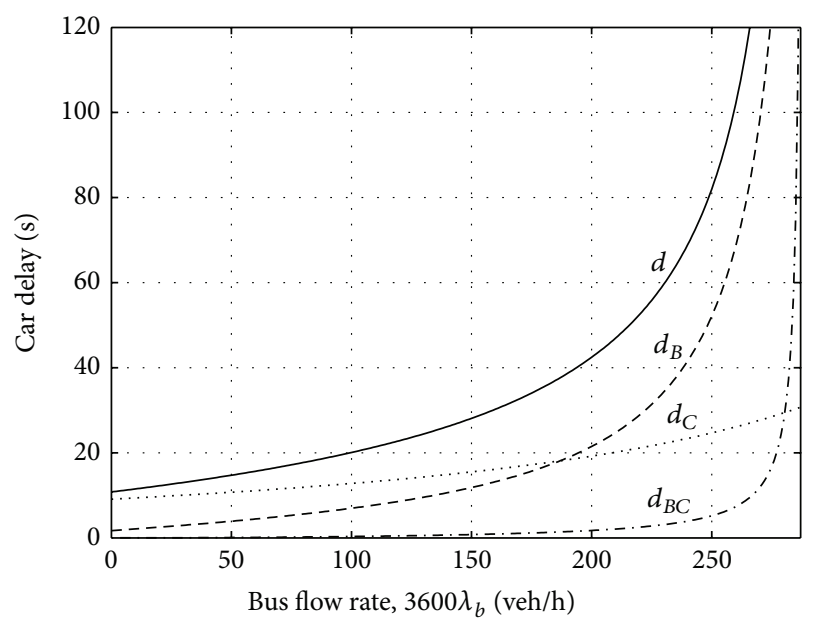

Figure 7: Effects of bus stream on car delay, $\lambda_{n}=0.6 \mathrm{veh} / \mathrm{s}, \lambda_{c}=$ $2 / 9 \mathrm{veh} / \mathrm{s}$, and $s_{b}=25 \mathrm{~s}$.

are consistent with observed phenomena that car delays are very long under high flow and slow speed conditions. In this case, most drivers would give way to bicyclists merging into the motorized lane as bicycles have advantages of small size, light weight, flexible action, and so on [12].

\section{Conclusion}

Delay time to cars at a bus stop with mixed traffic flow is investigated on the basis of queuing theory and probability theory. Bus stream system can be represented by an $\mathrm{M} / \mathrm{M} / \mathrm{k}$ queue. Meanwhile, the conflict between different streams at the stop can be described by the advanced Markovian model with no priorities but unequal service rates for customers. The delay resulting from following the slower bicycles can be obtained by the joint distribution of bivariate continuous random variable. The analysis shows that both bus stream and bicycle stream have significant effects on car delay. Car delay near the stop under mixed traffic condition is the function of 
three types of traffic streams with buses, cars, and bicycles. At bus volumes above approximately 200 vehicles per hour, the curbside stop design is unreasonable because of the long car delays. Therefore, in this case, it can be replaced by the bus bay design. The proposed model may be applicable to design and operational analysis of bus stops in other Asian developing countries.

Although this study has given valuable insights into car delay at the bus stop with mixed traffic flow, possible further research work is suggested. Firstly, we assume that bicycles have no priority over cars when bus berths at the stop in this paper. However, field observations show that sometimes a few bicyclists or drivers politely allow others to proceed. Bunker and Troutbeck [17] studied minor stream delays at a limited priority freeway merge. We need to present a new delay model which assumes limited priority for different streams near bus stops. In addition, the plan and design problems of bus stops with mixed traffic flow should be further researched.

\section{Acknowledgments}

This work was supported by the National Basic Research Program of China (Grant no. 2012CB725401), National Natural Science Foundation of China (Grants nos. 70901005, 71131001), and Fundamental Research Funds for the Central Universities (Grant no. 2013JBM043).

\section{References}

[1] Federal Transit Administration, Transit Cooperative Research Program Report 19: Guidelines for the Location and Design of Bus Stops, Transportation Research Board, Washington, DC, USA, 1996.

[2] W. Y. Szeto and Y. Z. Wu, "A simultaneous bus route design and frequency setting problem for Tin Shui Wai, Hong Kong," European Journal of Operational Research, vol. 209, no. 2, pp. 141-155, 2011.

[3] Á. Ibeas, L. Dell'Olio, B. Alonso, and O. Sainz, "Optimizing bus stop spacing in urban areas," Transportation Research E, vol. 46, no. 3, pp. 446-458, 2010.

[4] W. H. Gu, Y. W. Li, M. J. Cassidy, and J. B. Griswold, "On the capacity of isolated, curbside bus stops," Transportation Research B, vol. 45, no. 4, pp. 714-723, 2011.

[5] X. B. Yang, B. F. Si, and M. Huan, "Mixed traffic flow modeling near Chinese bus stops and its applications," Journal of Central South University, vol. 19, no. 9, pp. 2697-2704, 2012.

[6] S. C. Wong, H. Yang, W. S. A. Yeung, S. L. Cheuk, and M. K. Lo, "Delay at signal-controlled intersection with bus stop upstream," Journal of Transportation Engineering, vol. 124, no. 3, pp. 229-234, 1998.

[7] R. Fernández, "Modelling public transport stops by microscopic simulation," Transportation Research C, vol. 18, no. 6, pp. 856868, 2010.

[8] T. Q. Tang, Y. Li, and H. J. Huang, "The effects of bus stop on traffic flow," International Journal of Modern Physics C, vol. 20, no. 6, pp. 941-952, 2009.

[9] R. Z. Koshy and V. T. Arasan, "Influence of bus stops on flow characteristics of mixed traffic," Journal of Transportation Engineering, vol. 131, no. 8, pp. 640-643, 2005.
[10] D. H. Wang, T. J. Feng, and C. Y. Liang, "Research on bicycle conversion factors," Transportation Research A, vol. 42, no. 8, pp. 1129-1139, 2008.

[11] H. W. Guo, W. H. Wang, W. W. Guo, X. B. Jiang, and H. Bubb, "Reliability analysis of pedestrian safety crossing in urban traffic environment," Safety Science, vol. 50, no. 4, pp. 968-973, 2012.

[12] X. B. Yang, Z. Y. Gao, X. M. Zhao, and B. F. Si, "Road capacity at bus stops with mixed traffic flow in China," Transportation Research Record, vol. 2111, pp. 18-23, 2009.

[13] X. B. Yang, Z. Y. Gao, B. F. Si, and L. Gao, "Car capacity near bus stops with mixed traffic derived by additive-conflict-flows procedure," Science China Technological Sciences, vol. 54, no. 3, pp. 733-740, 2011.

[14] S. Teply, M. I. Abou-Henaidy, and J. D. Hunt, "Gap acceptance behavior: aggregate and logit perspective," Traffic Engineering and Control, vol. 38, no. 9, pp. 474-482, 1997.

[15] A. Polus and S. Shmueli, "Analysis and evaluation of the capacity of roundabouts," Transportation Research Record, vol. 1572, pp. 99-104, 1997.

[16] J. Medhi, Stochastic Models in Queueing Theory, Academic Press, San Diego, Calif, USA, 2nd edition, 2002.

[17] J. Bunker and R. Troutbeck, "Prediction of minor stream delays at a limited priority freeway merge," Transportation Research B, vol. 37, no. 8, pp. 719-735, 2003. 


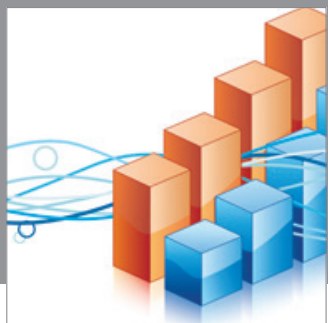

Advances in

Operations Research

mansans

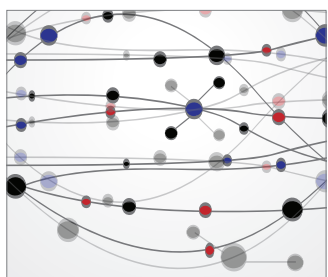

The Scientific World Journal
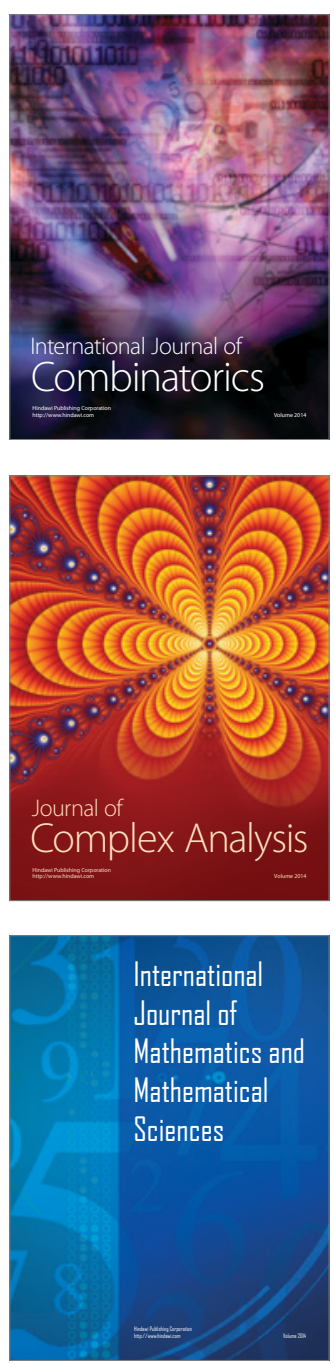
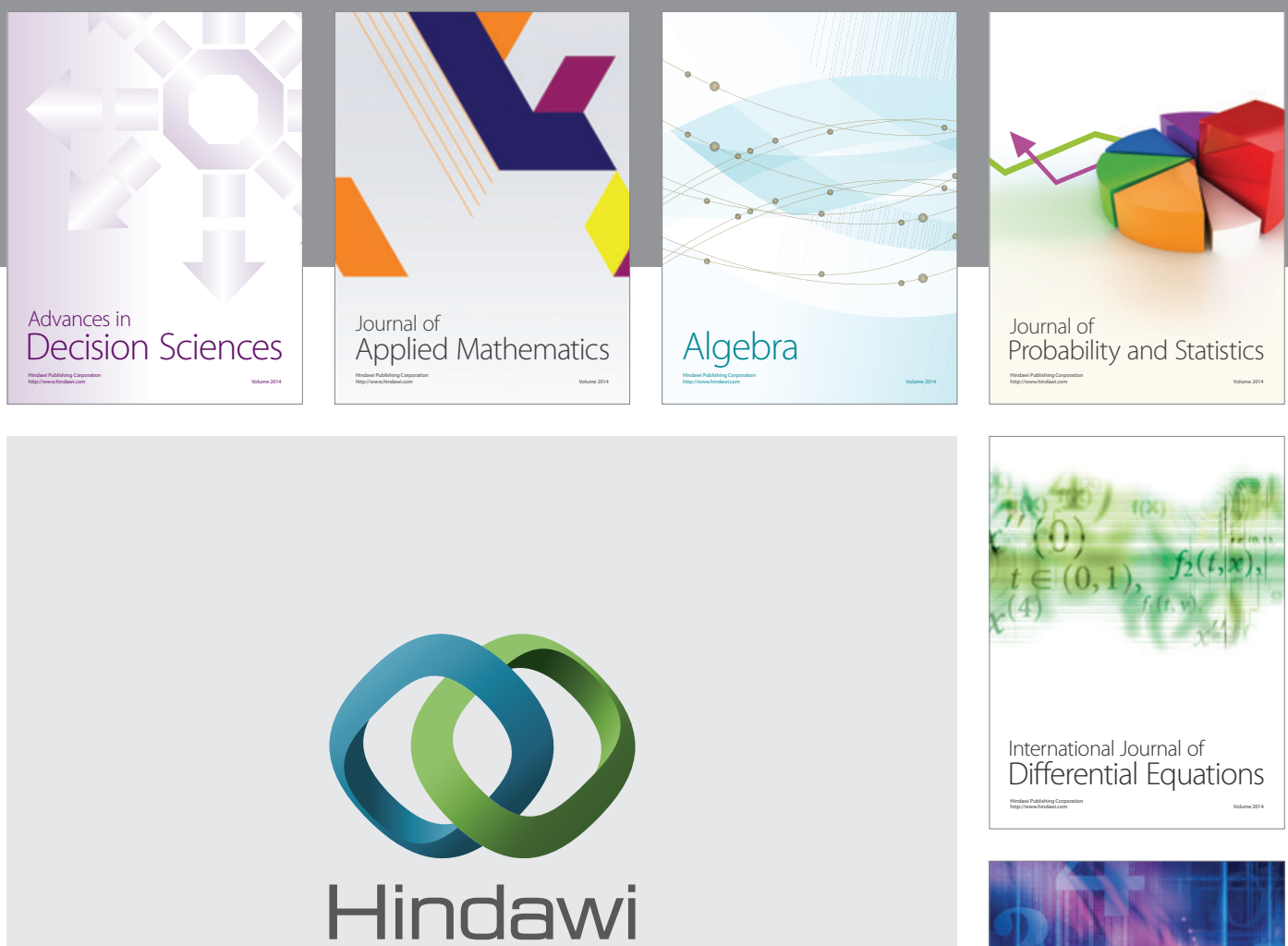

Submit your manuscripts at http://www.hindawi.com
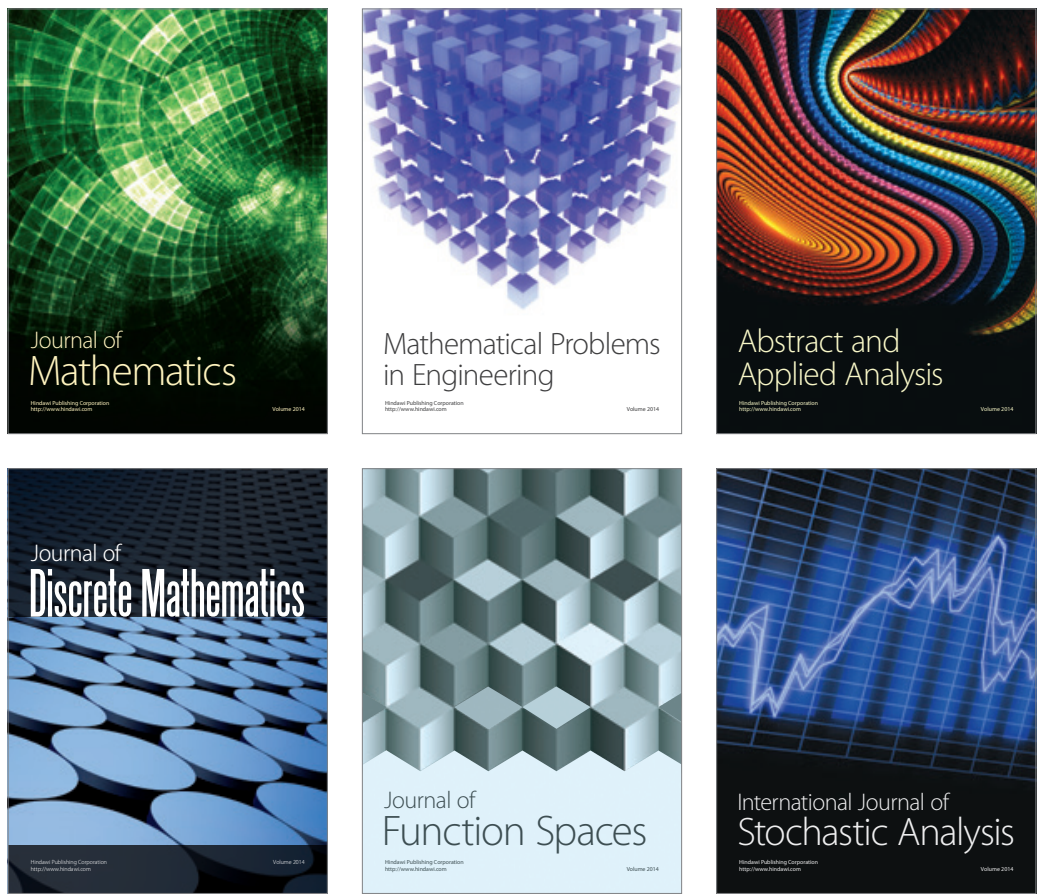

Journal of

Function Spaces

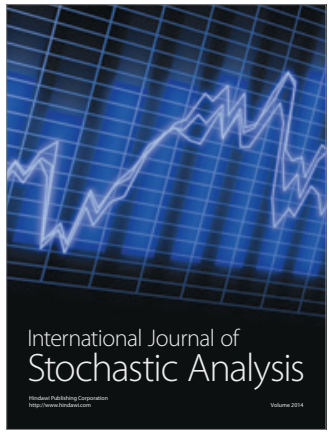

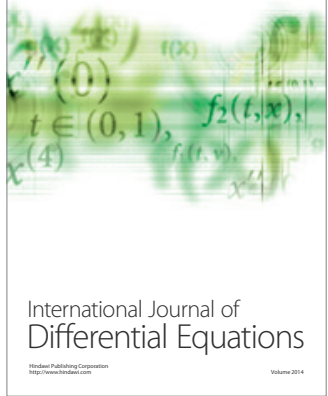
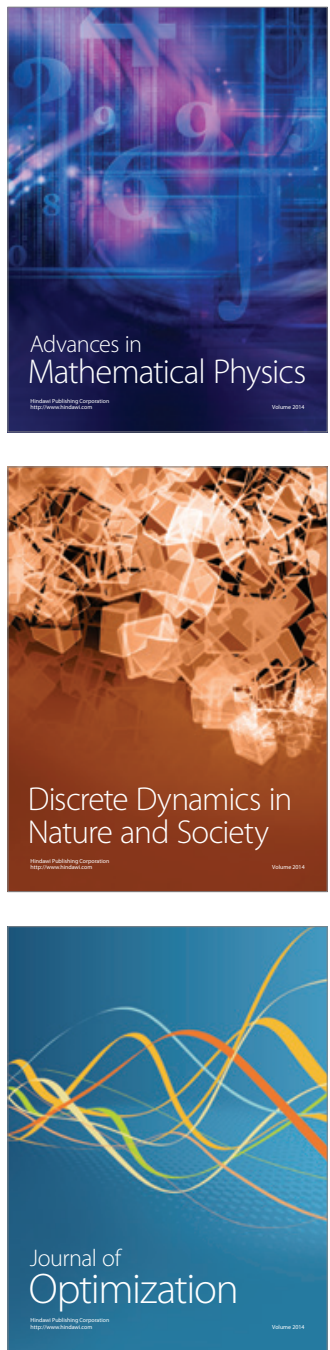